\title{
1
}

\section{Porous SiC Preparation, Characterization and Morphology}

\author{
Y. $\mathrm{Ke}^{1}$, Y. Shishkin ${ }^{2}$, R.P. Devaty ${ }^{1}$ and W.J. Choyke ${ }^{1}$ \\ ${ }^{1}$ Department of Physics and Astronomy, University of Pittsburgh, \\ Pittsburgh, PA 15260, USA \\ ${ }^{2}$ Caracal, Inc., 611 Eljer Way, Ford City, PA 16226, USA
}

\subsection{INTRODUCTION}

The production and investigation of porous semiconductors began in the 1950s at Bell Laboratories with work by Uhlir Jr [1] and Turner [2] on Si and Ge and at the Westinghouse Research Laboratories by Faust Jr [3] for SiC. Technological interest began with the discussion of the process of insulation by porous oxidized Si in the 1970s [4]. Interest in porous Si exploded in 1990 when Canham [5] at RSRE in the UK discovered that highly porous layers of $\mathrm{Si}$ excited by a blue laser emit readily visible red light at room temperature. The origin of this above band gap luminescence was a topic of heated controversy for several years. We became interested in the possibility of blue shifted luminescence into the ultraviolet (UV) in porous $\mathrm{SiC}$, and began to collaborate with Joseph Shor and colleagues, who were actively investigating electrochemical etching

Porous Silicon Carbide and Gallium Nitride: Epitaxy, Catalysis, and Biotechnology Applications Edited by Randall M. Feenstra and Colin E.C. Wood

(C) 2008 John Wiley \& Sons, Ltd 
of $\mathrm{SiC}$ at Kulite Semiconductor products and Columbia University [6]. They successfully fabricated porous $\mathrm{SiC}[7,8]$, but initial cathodoluminescence results [9] in 1994 did not convincingly reveal the desired effect. Further low temperature photoluminescence and cathodoluminescence measurements did not resolve this issue. However, we [10] found a dramatic effect of porosity on the reststrahl band of thick p-type $6 \mathrm{H}-\mathrm{SiC}$ layers observed using room temperature infrared (IR) reflectance. A simple effective medium model, chosen to incorporate the porous morphology, accounted for the observed spectral features. Subsequently, these effects have been observed in several other porous polar semiconductors and explained using similar models. Spanier and Herman [11] published further work on the IR reflectance of porous SiC. We ceased our optical investigations of porous $\mathrm{SiC}$ in 1995.

Our interest in porous $\mathrm{SiC}$ was renewed in 2000, based on some interesting work in the intervening years, which has been reviewed by Saddow et al. [12]. We set up a laboratory for fabricating porous SiC by (photo)electrochemical etching. We discovered (or rediscovered, in some cases) about ten distinct morphologies in $4 \mathrm{H}-, 6 \mathrm{H}-$ and $3 \mathrm{C}-\mathrm{SiC}$, as well as $\mathrm{GaN}$ layers [13]. This chapter focuses on the two morphologies we have investigated most thoroughly: the layered triangular and the nanocolumnar morphologies. For each case, we discuss the fabrication and characterization, followed by discussion of the formation mechanism.

\subsection{TRIANGULAR POROUS MORPHOLOGY IN n-TYPE 4H-SiC}

The 'triangular' porous morphology may be manufactured from highly doped hexagonal Si-face $4 \mathrm{H}-\mathrm{SiC}$ by photoelectrochemical etching (PECE) [14]. A small number of Si-face $6 \mathrm{H}-\mathrm{SiC}$ samples was also studied and revealed no significant difference from $4 \mathrm{H}-\mathrm{SiC}$. The observed morphology is explained based on the crystallography and a model for the semiconductor/electrolyte interface.

\subsubsection{Crystal Anodization}

The electrochemical etching, with the intent of making the etched semiconductor porous, is performed in the anodic regime, so that positive charge is collected at the semiconductor-electrolyte interface. In order to increase the speed of the anodization process, electrical bias is applied to the sample through ohmic contacts. To protect the ohmic contacts on the 
back as well as the edges during the anodization procedure, the samples are masked using Apiezon black wax. The experiments described here were performed in aqueous $5 \%$ HF solution mixed with ethanol in the ratio HF:ethanol = 1:1 (measured by weight).

There are two ways to produce a substantial anodic current. One way is to apply a large positive, or reverse, bias so that dielectric breakdown occurs and electrons from the ions in the electrolytic solution tunnel into the semiconductor. Voltages may range from 10 up to $100 \mathrm{~V}$. The resultant structures are dendritic, sinuous, and columnar. The other way is to apply a moderate reverse bias, from 1 to $3 \mathrm{~V}$, accompanied by UV light illumination (from which the term photoelectrochemical etching originates). The UV light of above photon band gap energy generates electron-hole pairs. The electrons are swept away by the electric field into the bulk of the semiconductor, while the holes rush to the surface where they participate in the electrochemical reaction.

To make porous $4 \mathrm{H}-\mathrm{SiC}$ of triangular morphology, we employed the second approach. As a UV light source one can use a Hg arc lamp. Filters and dichroic mirrors are recommended to attenuate visible and IR components of the radiation from the source. The UV light intensity was $\sim 160 \mathrm{~mW} / \mathrm{cm}^{2}$ as measured by a thermopile-type power meter. The electrochemical etching is conducted in a three-electrode cell where the $\mathrm{SiC}$ crystal serves as the working electrode. The current is controlled either potentiostatically (constant potential) or galvanostatically (constant current) with a potentiostat/galvanostat. In the potentiostatic mode, the voltage applied to the cell should not exceed $3 \mathrm{~V}$. When the galvanostatic mode was used, the fixed current density ranges from 1 to $5 \mathrm{~mA} / \mathrm{cm}^{2}$ so that the corresponding applied voltage would not exceed about $3 \mathrm{~V}$ in order to minimize breakdown effects.

After anodization, samples are carefully removed from the bath and cleaned in acetone to remove the black wax mask. As an alternative, trimethylchloroethylene may be used. Plan-view and cross-sectional imaging is performed by scanning electron microscopy (SEM). The 8-bit gray scale [intensity ranges from 0 (black) to 255 (white)] digital SEM pictures are then processed by imaging software to obtain the pore size and porosity. The porosity is estimated from cross-sectional images by integrating the area occupied by the pores.

\subsubsection{Description of the Porous Structure}

Figure 1.1 shows a series of cross-sectional SEM images of porous $4 \mathrm{H}$ $\mathrm{SiC}\left(n \sim 6 \times 10^{18} \mathrm{~cm}^{-3}\right)$ taken at different depths from about 5 to $55 \mu \mathrm{m}$ 

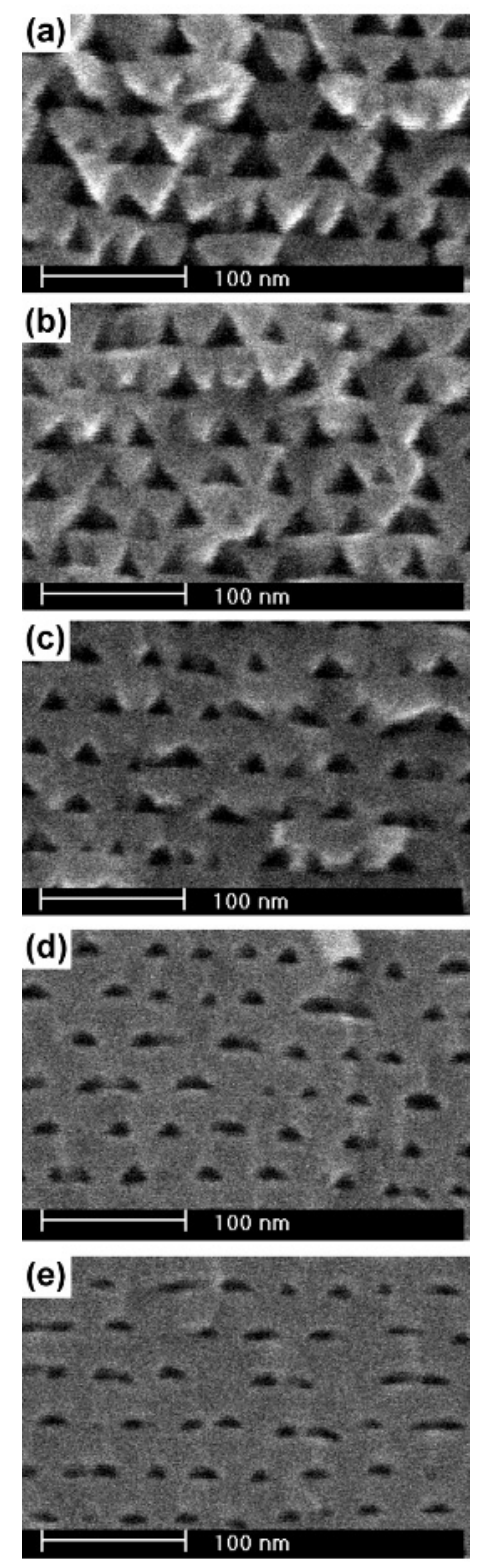

Figure 1.1 Sequence of cross-sectional images of a $56 \mu \mathrm{m}$ thick porous vicinal Siface $4 \mathrm{H}-\mathrm{SiC}$ sample of triangular morphology. In the sequence, (a) corresponds to the top of the film, (e) to the bottom. The distance $d$ from the front surface and the corresponding porosity $P$ are: (a) $d=5 \mu \mathrm{m}, P=0.28$; (b) $d=15 \mu \mathrm{m}, P=0.23$; (c) $d$ $=30 \mu \mathrm{m}, P=0.16$; (d) $d=40 \mu \mathrm{m}, P=0.12$; (e) $d=55 \mu \mathrm{m}, P=0.08$. Reproduced from Y. Shishkin et al., J. Appl. Phys., 96(4), 2311-2322. Copyright (2004), the American Institute of Physics 
below the surface. The morphology seen on Figure 1.1 was obtained in $8^{\circ}$-offcut $\mathrm{Si}$-face $4 \mathrm{H}$-SiC porous samples made by UV-photoassisted electrochemical etching at a voltage of $+3 \mathrm{~V}$. The particular surface exposed to the imaging by fracturing the sample is the $(1 \overline{2} 10)$ plane. The sample is organized into a stack of planar layers. When viewed from the $\langle 1 \overline{2} 10\rangle$ direction, a tilt of about $8^{\circ}$ of the layered structure relative to the normal to the front surface is seen (image not shown). A similar layering of pores is seen also in $3.5^{\circ}$-offcut $6 \mathrm{H}-\mathrm{SiC}$.

In photoelectrochemically etched n-type crystals, the porosity is rarely uniform throughout the porous layer. The porosity is largest at the surface and gradually decreases with depth. This is illustrated in Figure 1.1. As can be seen, not only the porosity changes but also there is a distinctive development of the triangular pore shape. Figure 1.1(e), taken near the interface between the porous layer and the substrate, shows that small oblong channels form in an orderly fashion. They are arranged in an array with rows separated by about $30 \mathrm{~nm}$ with the distance between pores also about $30 \mathrm{~nm}$ on average. The pore width at this stage is $5-10 \mathrm{~nm}$. A porous film produced under similar conditions for $2 \mathrm{~min}$, whose thickness was later measured to be $3 \mu \mathrm{m}$, exhibited only structure resembling Figure 1.1(e). This indicates that Figure 1.1(e), taken at the bottom of a 56 $\mu \mathrm{m}$ thick porous film (etched for $5 \mathrm{~h}$ ), portrays the initial stage of the triangular pore development.

In order to estimate the porosity, the pixels of the gray-scale images are thresholded so that those corresponding to a pore on the image are assigned the value 0 (black), and the pixels corresponding to the solid the value 1 (white). The total area occupied by the black pixels $S_{\text {black }}$ can be calculated [15]. Then the porosity $P$ is estimated by taking the ratio of $S_{\text {black }}$ to the total area of the cross-sectional image. Here, it is assumed that the areal porosity for a particular cross-section equals the volume porosity for the channel pore structure. For Figure 1.1(e), the porosity $P$ obtained by this method is 0.08 .

As the anodization process continues, the channels quickly pierce the crystal in directions perpendicular to the $c$-axis. The non-basal plane walls of every channel begin to flatten out [Figure 1.1(d)] to follow crystallographic planes tilted at about $60^{\circ}$ relative to the basal plane. One can clearly distinguish triangular shapes. Figure 1.1(b) and Figure 1.1(c) represent the continuing pore development as the channel walls become more distinct quasi-equilateral triangles. Their characteristic shape suggests anodization anisotropy, i.e. not all the directions in the crystal are equivalent in terms of the PECE. The tendency of the pores not to propagate normal to the [0001] direction suggests that the (0001) basal plane 


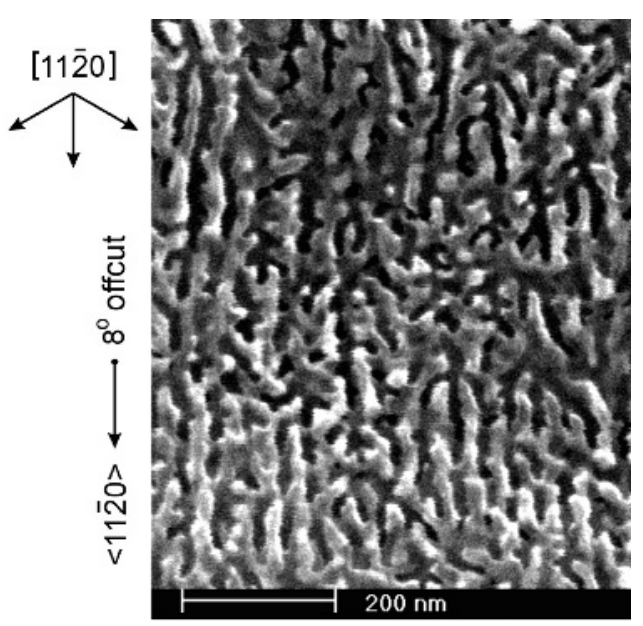

Figure 1.2 Plan view image of a $4 \mathrm{H}-\mathrm{SiC}$ Si-face sample, off-cut $8^{\circ}$ towards [1210], photoelectrochemically etched to obtain the triangular porous morphology. About $2 \mu \mathrm{m}$ of material was removed by RIE prior to imaging. The exposed channels apparently propagate preferably along $\langle 1 \overline{2} 10\rangle$ directions. Reproduced from Y. Shishkin et al., J. Appl. Phys., 96(4), 2311-2322. Copyright (2004), the American Institute of Physics

possesses an etch-stop character. The largest triangles are on Figure 1.1(a) corresponding to the top of the porous film. Here, the porosity $P$ is estimated to be about 0.28 . At this point we note that the experiments have shown so far no, or very little, porosity gradient in p-type $\mathrm{SiC}$ in which the etching is performed without light assistance [16].

Figure 1.2 is a plan view SEM image of a porous sample similar to the one shown in Figure 1.1 but processed for $15 \mathrm{~min}$ by reactive ion etching (RIE) with $\mathrm{SF}_{6}$ plasma to perform the plan view SEM analysis. Figure 1.2 shows how a vicinal basal plane appears with roughly $2 \mu \mathrm{m}$ removed by RIE. Compared with the non-RIE surface, which had just occasional circular openings, one can now see channels which tend to propagate parallel to the basal plane. The channels branch out, wriggle, and occasionally intersect each other. There seems to be a slight preference in the channel propagation along $\langle 1 \overline{2} 10\rangle$ directions, which are indicated by the arrows in Figure 1.2. The particular [1 $\overline{2} 10]$ direction towards which the $8^{\circ}$ miscut was made is indicated.

If the C-face $(000 \overline{1})$ is anodized, conical pores with triangular crosssections are observed, but the pores are not arranged into arrays of planes (images not shown). The conical pores intersect, forming an overall spongy network. Even though their typical size is very similar to that 


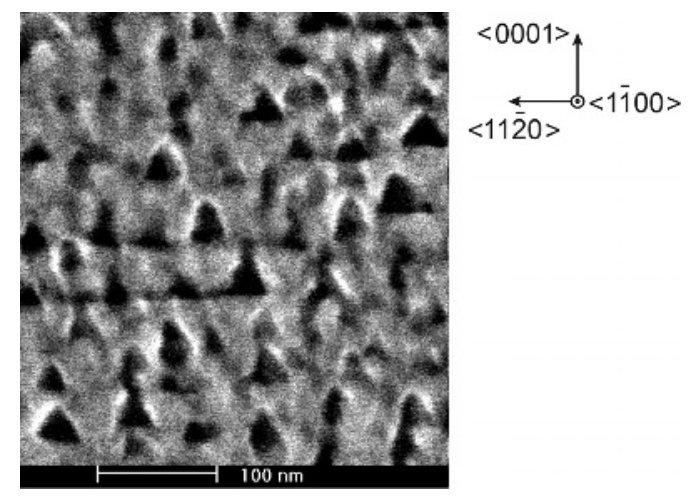

Figure 1.3 Plan view of a (1 $\overline{1} 00)$-oriented porous $4 \mathrm{H}-\mathrm{SiC}$ sample. 'Triangles' on the surface are about the same size as the 'triangles' seen on cross-sectional SEM images of the vicinal (0001) samples. Reproduced from Y. Shishkin et al., J. Appl. Phys., 96(4), 2311-2322. Copyright (2004), the American Institute of Physics

of the triangular channels, it is clear that Si-terminated and C-terminated surfaces etch differently.

The observed differences in the etching of (0001) and (000) crystal faces suggest etching experiments on non-basal planes. In these cases the electric field is applied parallel to the basal plane. Figure 1.3 shows an example of a front surface of a (1100) $4 \mathrm{H}-\mathrm{SiC}$ sample after UV photoassisted anodization. The front surface of an etched $(1 \overline{2} 10)$ sample looks similar within the resolution limits of the SEM. The surface morphology is described by layered triangular pits of average size of $30-40 \mathrm{~nm}$ observed all over the surface. The fact that the surface pits have a defined geometrical shape as well as their self-regulating character again point out anodization anisotropy.

The non-basal samples were also sectioned for analysis. Figure 1.4 shows an example for which a $4 \mathrm{H}-\mathrm{SiC}(1 \overline{1} 00)$ oriented sample has been fractured to expose the $(1 \overline{2} 10)$ plane. The crystal is seen to be divided into $35-50 \mathrm{~nm}$ thick layers. Each layer contains triangular cavities $30-40 \mathrm{~nm}$ in size. When the image in Figure 1.4 is rotated $90^{\circ}$ clockwise, a similarity to the cross-sectional images of the (0001) etched samples can be seen. The size and the shape of the triangular pores and their general planar layout on the fractured surface are identical. The similarity is striking given the fact that during etching the electric field is applied parallel to the basal plane and consequently perpendicular to the field applied to etch the sample in Figure 1.1. 


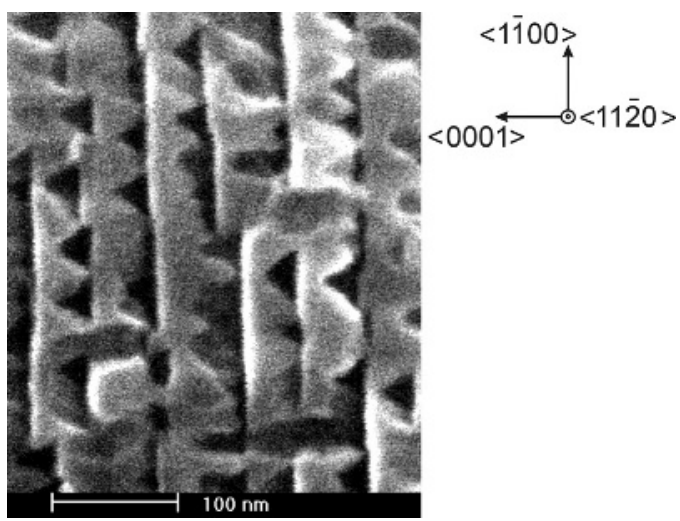

Figure 1.4 Cross-sectional image of a porous (1100) $4 \mathrm{H}-\mathrm{SiC}$ sample. The surface shown in the image is a $\{1 \overline{2} 10\}$ plane. When rotated, it resembles the cross-sectional images of vicinal Si-face samples. Reproduced from Y. Shishkin et al., J. Appl. Phys., 96(4), 2311-2322. Copyright (2004), the American Institute of Physics

Figure 1.5 shows an example for which a $4 \mathrm{H}-\mathrm{SiC}(1 \overline{1} 00)$ oriented sample has been fractured to expose a basal plane. The channeled structure of the pores can still be discerned. The arrows next to the figure show the $\langle 1 \overline{2} 10\rangle$ directions. There are no (or very few) channels aligned with $\langle 1 \overline{1} 00\rangle$, suggesting that $\langle 1 \overline{2} 10\rangle$ are the primary directions along which

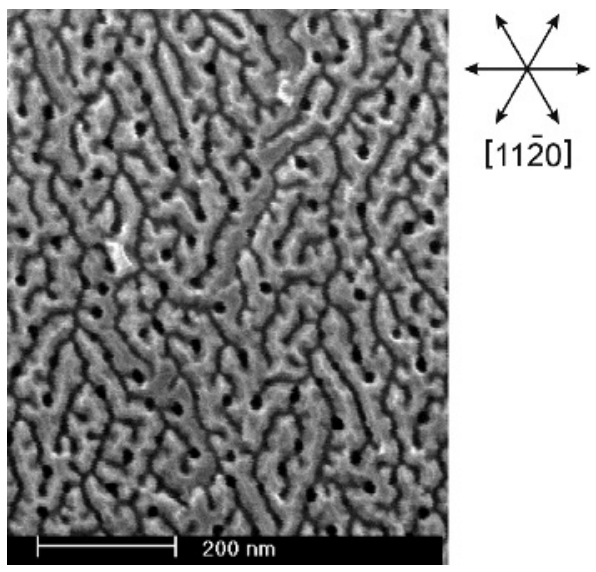

Figure 1.5 Cross-sectional image of a porous (1100) $4 \mathrm{H}-\mathrm{SiC}$ sample. The surface shown in the image is a basal plane. Compare the wormy character of the pore channels with Figure 1.2. Reproduced from Y. Shishkin et al., J. Appl. Phys., 96(4), 2311-2322. Copyright (2004), the American Institute of Physics 
the pores prefer to propagate. Comparing the result with Figure 1.2, where we were able to look at the basal plane by means of RIE etching off the top layer, we again conclude that pores do not propagate well along the $c$-axis. Moreover, the direction of the electric field applied during the experiment does not seem to be a factor in the pore formation in the investigated voltage/current regime.

\subsubsection{Model of the Morphology}

\subsubsection{Pore Dimensions and Porosity}

One of the arguments used in the porous silicon literature to explain the typical dimension of the structures states that the pore formation is governed by the width of the space charge region formed near the semiconductor/electrolyte junction (see, for example, [17]). The argument seems to be very plausible as it works well for the mesoporous structures (10-100 nm pore size) obtained from n-type Si. The model has also been proposed for porous SiC [18] and elaborated in Shishkin et al. [14].

The width of the space charge (or, depletion) layer $L_{s c}(\mathrm{~cm})$ is determined by solving the Poisson equation with appropriate boundary conditions. For the case of an n-type semiconductor with a planar surface, such that $n \approx N_{d}$, and $N_{d} \gg N_{a}$, the charge density is approximated as $\rho \approx e n$. One obtains:

$$
L_{s c}=\sqrt{\frac{2 \varepsilon_{s c} \varepsilon_{0} \phi_{s c}}{e n}}
$$

At the potential $\phi_{s c}=3 \mathrm{~V}$, the width of the space charge region when the free carrier concentration is $n=6 \times 10^{18} \mathrm{~cm}^{-3}$ is calculated to be $L_{s c} \sim 30 \mathrm{~nm}\left(\varepsilon_{s c}\right.$ is assumed $\left.\sim 10\right)$. As we showed in Figure 1.1(e), the average distance between the newly formed channels in the triangular pore structure obtained by PECE is $30-35 \mathrm{~nm}$. The calculated value for $L_{s c}$ then may be interpreted as a typical distance which determines the selfregulatory character of the lateral (i.e. parallel to the basal plane) pore formation process. Piercing the crystal, the channels align themselves into a network in such a way that for each given channel the onset of another channel next to it occurs at a distance where the electric field produced by the band bending vanishes, i.e. where the depletion layer ends. Such pore initiation-alignment processes happen both vertically and horizontally. 
Depleted of charge carriers, the interpore spacing of a newly formed porous structure exhibits semi-insulating properties with the Fermi level of the semiconductor pinned to the surface energy levels close to the middle of the band gap [18]. Such pinning results in a different distribution of the potential at the interface as compared with a planar semiconductor/electrolyte junction without surface states. In particular, a substantial potential drop in the so called Helmholtz region of the electrolyte occurs, which has a few nanometers thickness and is adjacent to the sample surface. The field in the semiconductor then is expected to become lower, decreasing the band bending at the surface and slowing down the charge transfer through the interface. In this regard, the stages of the pore formation can be deduced indirectly from the current versus time dependence recorded over the course of the PECE experiment. In Figure 1.6, the etching starts in the $\mathrm{mA} \mathrm{cm}^{-2}$ range, which is due to the rapid pore initiation process which manifests itself in the fact that one can obtain a porous film a few micrometers thick in $1 \mathrm{~min}$. The cross-sectional SEM image of such a film shows small 'shapeless' channels similar to what is seen in Figure 1.1(e). A rough estimate gives the total internal surface area of the pores at this stage to be 50-60 times larger than the front surface area of the sample. This turns out to be enough to substantially increase the number of collected holes. The current density quickly rises to a value

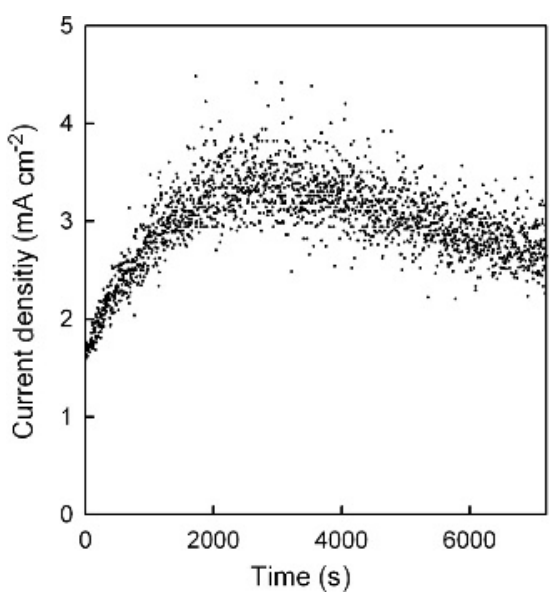

Figure 1.6 Current density vs time for an n-type 4H-SiC sample etched at $3 \mathrm{~V}$ for 2 h. The sample area is $\sim 0.45 \mathrm{~cm}^{2}$. Integration of the curve provides the total charge transferred during the course of the reaction leading to the estimate of porosity $P \sim 0.2$. Reproduced from Y. Shishkin et al., J. Appl. Phys., 96(4), 2311-2322. Copyright (2004), the American Institute of Physics 
an order of magnitude higher than its initial value on a flat (nonporous) surface.

As seen in Figure 1.6, the current density reaches its maximum value of about $3 \mathrm{~mA} \mathrm{~cm}^{-2}$ in approximately $30 \mathrm{~min}$, and then gradually drops, indicating a decreasing rate of dissolution. Such a decrease in the anodic current density for $\mathrm{SiC}$ has also been reported in the literature $[8,19]$. Despite the noisy character, the current density $j(t)$ dependence in Figure 1.6 may be used to calculate the integrated charge $Q_{i n t}=\int i(t) \mathrm{d} t=A \int j(t) \mathrm{d} t$ transferred during the course of the reaction. Knowledge of $Q_{\text {int }}$ allows one to estimate the average porosity $\bar{P}=V_{p} / V$. The total volume of the semiconductor $V$ is simply the product of the front surface area $A$ and the film thickness $d$. The total 'void' volume produced by dissolution is $V_{p}=N \cdot V_{0}$ where $N$ is the number of Si-C pairs dissolved and $V_{0}$ is the volume of a single $\mathrm{Si}-\mathrm{C}$ pair in the $\mathrm{SiC}$ crystal lattice. $V_{0}$ is obtained from the lattice constants of $4 \mathrm{H}-\mathrm{SiC}: a=3.081 \AA, c / 4=2.52 \AA$ [20]. $N$ is $Q_{i n t} / e \gamma$, where $\gamma$ is the number of holes required to dissolve one Si-C pair. Combining all the terms, we write

$$
\bar{P}=\frac{V_{p}}{V}=\frac{V_{0} Q_{i n t} / e \gamma}{A d}=\frac{V_{0} \int j(t) \mathrm{d} t}{e d \gamma} .
$$

The gravimetric measurements for $4 \mathrm{H}$ n-type $\mathrm{SiC}$ show that $\gamma$ is very close to 7 , within $1 \%$ accuracy [14].

When the $j(t)$ curve (see Figure 1.6) is integrated, one gets roughly $22.3 \mathrm{C} \mathrm{cm}^{-2}$. Once the porous film thickness is determined from the cross-sectional SEM measurement $(d \sim 16 \mu \mathrm{m}$ for the sample in Figure 1.6), one can obtain the value of the average porosity for the porous planar/triangular morphology obtained in n-type $4 \mathrm{H}-\mathrm{SiC}$ using Equation (1.2). After inserting all the numbers, the estimated average porosity is $\bar{P}=V_{p} / V \approx 0.20$.

SEM images have also been obtained for the above sample at 1, 4, 7, 10,14 and $16 \mu \mathrm{m}$ from the front surface. The resultant dependence of $P$ as a function of depth $d$ is shown in Figure 1.7. This $16 \mu \mathrm{m}$ thick sample has a clear porosity gradient which is reflected by the decreasing values of $P$ with the increasing of $d$. If fitted to an exponential,

$$
P(d)=P_{0}+a \mathrm{e}^{-b d},
$$

the constant $b$ can be determined (values of the offset $P_{0}$ are negligible.). For the sample in Figure 1.7, $b=880 \mathrm{~cm}^{-1}$. Making a similar analysis 


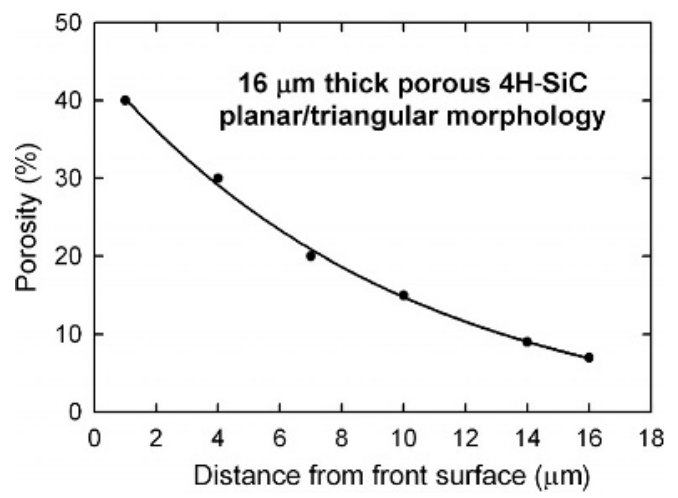

Figure 1.7 The porosity as a function of distance from the front surface of the sample. The porous layer was obtained in the run shown in Figure 1.6. The values of porosity are extracted from digital analysis of the SEM images. The solid line is a fit to Equation (1.3), which gives $P=21 \%$ for the average porosity. Reproduced from Y. Shishkin et al., J. Appl. Phys., 96(4), 2311-2322. Copyright (2004), the American Institute of Physics

for samples etched for shorter times (so that the photo-electrochemical action proceeds closer to the original, 'starting', surface) results in larger values of $b$. For longer etching times used to produce thicker films, the collection of holes, on average, takes place at larger depths where the longer wavelengths of the UV light, associated with lower absorption coefficient, penetrate more efficiently. Therefore, $b$ is a representative coefficient which takes into account the range of UV lines participating in the etching process.

The integration of the $P(d)$ function given by Equation (1.3) gives the average porosity of the porous layer. A value of 0.21 is obtained from the data in Figure 1.7 when integration is done from 0 to $d_{\max }=16 \mu \mathrm{m}$. Thus, the porosity estimated from SEM images is in good agreement with the average porosity calculated using the charge transfer $(P \sim 0.20)$.

\subsubsection{Model for the Pore Shape}

The base of each 'triangle' always lies in the basal plane whereas its sidewalls are oriented about $60^{\circ}$ relative to the base. This pore shape implies some etching relationship to the crystallographic planes of the original $\mathrm{SiC}$ single crystal.

Our SEM cross-sectional images show that the etching rate, defined as the thickness of a porous layer etched per unit time, is a factor of 
two larger for a $\{1 \overline{2} 10\}$ plane than for a $\{1 \overline{1} 00\}$ plane under identical conditions. This fact supports our observation deduced from Figure 1.2 that the $\{1 \overline{2} 10\}$ surface is less resistant to electrolytic attack. Qualitatively, this can be explained by the fact that, in hexagonal polytypes, $\{1 \overline{2} 10\}$ surfaces have a higher surface energy than the $\{1 \overline{1} 00\}$ surfaces [21]. It is reasonable to assume that those directions will be chosen for the pore growth which are easier to etch. The surfaces of low energy are the ones which 'survive' the etching and the channel pore patterns are formed.

Even though the etch rate difference between an a-face $\{1 \overline{2} 10\}$ and a p-face $\{1 \overline{1} 00\}$ provides the possibility for the channel formation, it is not sufficient to explain the triangular shape. Pores form by an oxidation of the surface silicon and carbon atoms with the immediate removal of the oxidized species into the electrolytic solution. Therefore, in order to investigate the issue of a particular plane's etching inertness, one has to make an assumption that the oxidation is substantially weaker on Si-rich surfaces of $\mathrm{SiC}$ as compared with the C-rich ones. The basis for this statement depends upon experiments on the oxidation of the Si- and C-faces of $\mathrm{SiC}$, in which the polar character of the silicon-carbon bond [22] was first suggested to be responsible. Muelhoff et al. [23] reported different oxidation rates for $(0001)$ and $(000 \overline{1})$, i.e. Si-rich and C-rich, surfaces for thermally oxidized $\mathrm{SiC}$. The oxidation of a Si-terminated surface proceeds at a much slower rate. Although the oxidation chemistry in aqueous environment is likely somewhat different from that reported in Muelhoff et al. [23], in which dry oxygen was used, the rate of oxidation for the $(000 \overline{1})$ surface is still larger by at least a factor of two than that of the (0001) surface when water vapor is used for oxidation. Consequently, the pore shape in a $\mathrm{SiC}$ crystal is greatly affected by the surface polarity. Since we have already shown the etch-stop character of the (0001) plane, a silicon-terminated surface, we propose that the surfaces which make up the sidewalls of our triangular channels are silicon-rich etch-stop surfaces also.

Let us now examine the lattice structure of $4 \mathrm{H}-$ and $6 \mathrm{H}-\mathrm{SiC}$ polytypes. Having established the preference for triangular pore propagation along $\langle 1 \overline{2} 10\rangle$ directions, the logical thing to do would be, looking along a $\langle 1210\rangle$ direction, to find those surfaces which are terminated with silicon atoms. Figure 1.8 shows a schematic view of a $4 \mathrm{H}-\mathrm{SiC}$ crystal lattice as seen from a $\langle 1 \overline{2} 10\rangle$ direction. The zigzagged lines connecting silicon atoms (lighter shaded dots) make up the surfaces of the walls forming a triangle. Two of the thick solid lines represent the $(\overline{1} 10 \overline{2})$ and $(1 \overline{1} 0 \overline{2})$ planes. We propose that, like the $(0001)$ surface, the $(\overline{1} 10 \overline{2})$ and $(1 \overline{1} 0 \overline{2})$ 


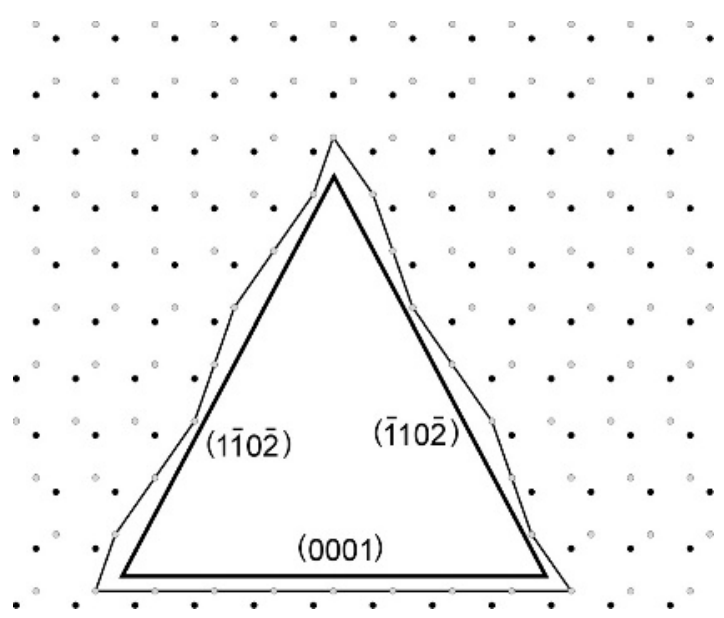

Figure 1.8 Schematic of the projection of the 4H-SiC crystal lattice as viewed from a $\langle 1 \overline{2} 10\rangle$ direction. The planes comprising the walls of the imaginative triangular channel are indicated. Light dots, silicon atoms; black dots, carbon atoms. Reproduced from Y. Shishkin et al., J. Appl. Phys., 96(4), 2311-2322. Copyright (2004), the American Institute of Physics

surfaces are also nominally terminated with silicon atoms. The counterparts $(\overline{1} 102)$ and $(1 \overline{1} 02)$ are then terminated with carbon atoms. An oxidation experiment conducted on $6 \mathrm{H}-\mathrm{SiC}$ using $(1 \overline{1} 03)$ and $(1 \overline{1} 0 \overline{3})$ surfaces, which are somewhat similar to the respective $(1 \overline{1} 02)$ and $(1 \overline{1} 0 \overline{2})$ surfaces in $4 \mathrm{H}-\mathrm{SiC}$, confirms the model [24]. Despite the lack of data on $4 \mathrm{H}-\mathrm{SiC}$, we are confident that silicon termination makes the $(\overline{1} 10 \overline{2})$ and $(1 \overline{1} 0 \overline{2})$ surfaces behave like etch-stops under anodization conditions, similar to the (0001) face. Therefore, the sides of each triangle are made of surfaces corresponding to the $\{1 \overline{1} 0 \overline{2}\}$ family of planes. This fact plus preferential pore propagation along the $\langle 1 \overline{2} 10\rangle$ directions create the necessary environment for the observed triangular-channeled structural pore shape.

Next we determine the angle of inclination $\theta$ of the triangular sides of the pores, assuming the $\{1 \overline{1} 0 \overline{2}\}$ planes to be the walls of these triangles. One way of doing this is to determine the components of the reciprocal lattice vector $\langle 1 \overline{1} 02\rangle$ in terms of the basis vectors of the real space $\hat{x}, \hat{y}$, and $\hat{z}[14]$ :

$$
\langle 1 \overline{1} 02\rangle=1 \cdot \vec{b}_{1}-1 \cdot \vec{b}_{2}+2 \cdot \vec{b}_{3}=-\frac{4 \pi}{a \sqrt{3}} \hat{y}+\frac{4 \pi}{c} \hat{z}
$$


Based on the components of the vector in the reciprocal lattice space the angle $\theta$ is found as:

$$
\tan \theta=\frac{4 \pi / a \sqrt{3}}{4 \pi / c}=c / a \sqrt{3}=1.889
$$

which gives $\theta=62.1^{\circ}$. The inclination of the $\{1 \overline{1} 0 \overline{2}\}$ plane is indeed very close to $60^{\circ}$, explaining why we observe the pore cross-sections as almost equilateral triangles. This result plus preferential pore propagation along the $\langle 1 \overline{2} 10\rangle$ directions, discussed earlier, create the necessary environment for the observed triangular-channeled pore structure.

Since it has been proposed that this surface is terminated with silicon atoms, it would be logical to assume that the surfaces corresponding to the $\{1 \overline{1} 02\}$ family of planes are terminated with carbon atoms. Nonbasal planes corresponding to pore surfaces are interesting candidates for boule growth experiments, electrical properties studies, and surface science investigations [24-26]. Already, low-energy electron diffraction (LEED) and Auger electron spectroscopy (AES) have revealed differences in the composition of the $(1 \overline{1} 02)$ and $(1 \overline{1} 0 \overline{2})$ surfaces of $4 \mathrm{H}-\mathrm{SiC}[26]$.

\subsection{NANO-COLUMNAR PORE FORMATION IN n-TYPE $6 \mathrm{H}-\mathrm{SiC}$}

The achievements of making controllable self-organized columnar porous alumina, porous $\mathrm{Si}$ and porous $\mathrm{InP}$ [27-32] raise the question whether we can obtain a similar porous structure in $\mathrm{SiC}$ by controlling the etching conditions. This section focuses on the fabrication of columnar porous SiC. The necessary etching conditions and formation mechanisms are also discussed.

\subsubsection{Experimental}

The experimental set-up and technique to fabricate columnar porous $\mathrm{SiC}$ are similar to the set-up used for triangular porous $\mathrm{SiC}$. But, since we need to apply a high voltage $(>10 \mathrm{~V})$ and use a relatively concentrated HF electrolyte, the PECE experiments are conducted in a two-electrode cell where a $\mathrm{SiC}$ crystal is used as the anode. Aqueous $10 \% \mathrm{HF}$ mixed with $5 \%$ ethanol (measured by weight) electrolyte is used. Voltage is applied 
across the two electrodes using a PAR 2273 Potentiostat/Galvanostat. Since front side illumination is needed to increase the hole concentration in the n-type semiconductor, a $1000 \mathrm{~W}$ Oriel Hg Xe Arc Lamp combined with optical filters is used to produce a flux of UV photons with intensity about $600 \mathrm{~mW} \mathrm{~cm}{ }^{-2}$ as measured by a thermopile-type light power meter.

Up to now, both $6 \mathrm{H}$ - and $4 \mathrm{H}-\mathrm{SiC}$ samples from single crystal, on axis, n-type nitrogen doped (range from $3 \times 10^{17}$ to $3 \times 10^{18} \mathrm{~cm}^{-3}$ ) wafers have been used for making the nano-columnar porous structure with the proper selected etching conditions. In this section, we focus on the etching of n-type $6 \mathrm{H}$ material doped about $1 \times 10^{18} \mathrm{~cm}^{-3}$. For comparison of porous structures, we set the etching times at $1 \mathrm{~h}$ in all experiments, unless specifically stated otherwise.

A Sartorius MC21S microbalance is used to measure the mass loss of the sample due to the PECE. Planar and cross-sectional scanning electron microscope imaging is then performed using a Philips XL30 FEG microscope at an operating voltage of $15 \mathrm{kV}$. The thickness of the porous structure is determined by the cross-sectional SEM measurement. To reveal the structure at some depth from the top surface, we use a Plasma-Therm 790 RIE system to remove a known thickness of the porous structure. In the RIE etching process, $20 \mathrm{mTorr}^{\mathrm{SF}_{6}}$ gas is used with $250 \mathrm{~W}$ power applied.

\subsubsection{Results}

Initial attempts to produce columnar porous $\mathrm{SiC}$ were performed on the Si-face by applying larger voltage $(>50 \mathrm{~V})$ or current density $(>50 \mathrm{~mA}$ $\mathrm{cm}^{-2}$ ). The columnar pore growth under these high field conditions is unstable, and the result was either a columnar macroporous $(\sim 1 \mu \mathrm{m}$ diameter) structure or a hybrid partial columnar structure (low volume fraction of large diameter columns penetrating dendritic nanoporous $\mathrm{SiC}$ ) [33]. In addition to the fact that the pore size is difficult to adjust, the macrocolumnar porous structure has high porosity $(>0.9)$ and thus is too fragile to be generally useful for practical applications.

Our research on the triangular porous morphology in $6 \mathrm{H}-\mathrm{SiC}$ indicates that the Si-face is an electrochemical etching resistant plane. Therefore, attempting columnar porous growth on the Si-face of $\mathrm{SiC}$ is essentially etching through an unfavorable crystalline face. Etching the Si-face therefore tends to favor branching rather than the development of long, straight columns. Unless an avalanche breakdown etching 


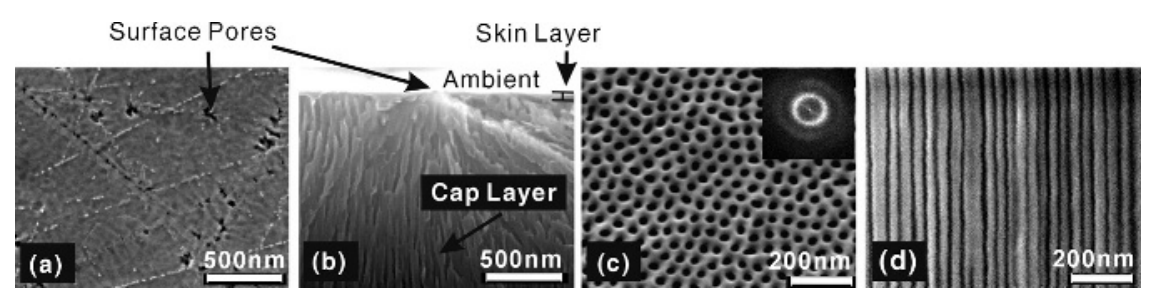

Figure 1.9 SEM images [34] of (a) original porous SiC surface after PECE, (b) early stage formation of columnar pore in cross-section, (c) porous surface structure $20 \mu \mathrm{m}$ below the original surface after $90 \mathrm{~min}$ of RIE (the inset shows the Fourier transform of a larger area of this picture), and (d) the self-ordered columnar porous structure below the cap layer in cross-section. Reproduced from Y. Ke, R.P. Devaty and W.J. Choyke, Self-ordered nanocolumnar pore formation in the photoelectrochemical etching of 6H SiC, Electrochem. Solid-State Lett., 10(7), K24-K27 (2007). Copyright 2007, with permission from The Electrochemical Society

condition (even locally) is achieved, the columnar pore growth will not happen. To achieve controllable columnar pore growth, electrochemical etching should be done on 'non-etch stop' faces. The fact that the C-face oxidizes much faster than the Si-face in wet oxidation [23] suggests that in another kind of oxidation - the photo-electrochemical etching process - the C-face is less likely to be an etch stop than the Si-face. This suggests that etching the C-face of a SiC crystal may favor the columnar pore formation.

When $20 \mathrm{~V}$ etching voltage is applied to a $\mathrm{C}$-face $\mathrm{SiC}$ sample, a uniform nano-columnar porous structure forms. Figure 1.9 shows SEM images of the formed columnar porous structure. From these images we can see that the pore morphology in the porous $\mathrm{SiC}$ is similar to those seen in porous $\mathrm{Si}, \mathrm{Al}_{2} \mathrm{O}_{3}$ and InP [27-32]. From our cross-sectional SEM images [Figure $1.9(\mathrm{~d})$ ], we estimate that the pore diameter is about $20 \mathrm{~nm}$ and the pore wall thickness is about $40 \mathrm{~nm}$. Within our SEM resolution, no detailed structure is seen on the pore walls. The pore walls are smooth. The $200 \mu \mathrm{m}$ long nano-columnar pores are generally straight and parallel.

The planar SEM image [Figure 1.9(a)] shows that the columnar pores do not form right at the surface. The cross-sectional SEM image of the ambient/porous interface [Figure 1.9(b)] indicates a $\sim 10 \mathrm{~nm}$ thick initial low porosity 'skin layer'. The surface pore nucleation tends to follow the scratch marks left by mechanical polishing and shows no particular order [35]. The areal density of the surface pores is far less than that of the columnar pores formed deeper in the bulk. The subsequent porous growth initiates from the surface pores. The pores are neither regular nor straight, and keep branching without following any particular crystalline 
direction. The regularity of the columnar pore lattice improves with increasing depth. An ordered columnar porous structure can be recognized about $10 \mu \mathrm{m}$ below the skin layer. For convenience, we call this $\sim 10 \mu \mathrm{m}$ thick transition porous structure the 'cap layer'.

To reveal the columnar porous structure and check the regularity of the porous pattern below the cap layer, we RIE the sample for $90 \mathrm{~min}$ and remove roughly $20 \mu \mathrm{m}$ of the porous structure from the top. A planar-view SEM image of the freshly exposed surface shows a semi-selforganized porous pattern [Figure 1.9(c)]. The Fourier transform inset shows a multiple ring structure. This suggests that the columnar pores fabricated so far do not have an orientational long range order. The columnar pores are, however, uniformly distributed in space and possess some short range regularity as a hexagonal pore lattice. The diameter of the pores is about $30 \mathrm{~nm}$ instead of $20 \mathrm{~nm}$ as seen on the cross-sections [Figure 1.9(d)]. We believe that the RIE process widens the openings of the columnar pores.

Based on the weight loss during the PEC process measured using the microbalance, the porosity is estimated to be 0.1 . Because the columnar pores are generally uniform throughout the porous layer, it is reasonable to assume that the columnar porous structure we obtain is comparable with a perfect hexagonal packed cylindrical pore lattice. For such a pore lattice, the porosity $P$ can be calculated using:

$$
P=\frac{2 \pi}{\sqrt{3}}\left(\frac{r}{D}\right)^{2}
$$

where $r$ is the radius of the columnar pore and $D$ is the distance between adjacent pore centers. According to the SEM images, the estimated values are $r \approx 10 \mathrm{~nm}$ and $D \approx 60 \mathrm{~nm}$, and the calculated porosity $P=0.1$, which agrees with the gravimetric porosity. This value is also similar to the porosity of self-organized porous alumina [30]. The reason for this particular porosity is discussed for the porous alumina, but remains unclear for columnar porous $\mathrm{SiC}$. Nevertheless, the mechanical strength associated with this low porosity makes this nano-columnar porous $\mathrm{SiC}$ a potentially useful nano-material for practical applications.

\subsubsection{Discussion}

After the fabrication of the columnar porous structure, to gain a deeper insight into the formation mechanism, we performed the following three 
comparison PECE experiments:

EXP. 1: C-face SiC etched under $20 \mathrm{~V}$ constant voltage conditions.

EXP. 2: Si-face $\mathrm{SiC}$ etched under $20 \mathrm{~V}$ constant voltage conditions.

EXP. 3: C-face $\mathrm{SiC}$ etched under $30 \mathrm{~mA} \mathrm{~cm}^{-2}$ constant current conditions.

To evaluate the photoelectrochemical reactions in these PECE processes, it is useful to calculate and compare the average number of holes ' $\gamma$ ' needed to etch away a single $\mathrm{SiC}$ pair. The agreement among different experiments points to a similar electrochemical process. $\gamma$ is calculated using the following expression:

$$
\gamma=N_{\text {holes }} / N_{S i C}=\frac{Q}{e} / \frac{V_{p}}{V_{0}}=\frac{Q}{e} / \frac{\Delta m}{\rho V_{0}}=\frac{Q \rho V_{0}}{e \Delta m} .
$$

Here, $Q$ is the total electric charge flowing through the sample during the experiment ( $Q$ is estimated by integrating the current-time curves), $e$ is the unit electric charge $\left(1.6 \times 10^{-19} \mathrm{C}\right), N_{\text {boles }}=Q / e$ is the total number of holes that flowed, $\Delta m$ is the mass loss and $\rho$ is the SiC mass density $\left(3.21 \mathrm{~g} \mathrm{~cm}^{-3}\right) . V_{p}$, the pore volume, which is the total volume of $\mathrm{SiC}$ pairs removed by the PECE process, is then $\Delta m / \rho . V_{0}$ is the volume of a $\mathrm{SiC}$ pair and can be calculated $\left(\mathrm{V}_{0}=20.7 \times 10^{-24} \mathrm{~cm}^{3}\right)$ using $6 \mathrm{H}-\mathrm{SiC}$ lattice constants [20].

In all three experiments, about seven holes (7.3 in EXP. 1; 6.9 in EXP. 2; 7.1 in EXP. 3) on average are needed for a single SiC pair to be removed. These results are also consistent with the hole number calculated for triangular porous $\mathrm{SiC}$ formation and the PECE reported by Shor and Kurtz [8]. This implies that all the photoelectrochemical reactions are similar, at least in the number of holes needed to etch each $\mathrm{SiC}$ pair. The different porous morphologies obtained here are unlikely to be related to different chemical reactions.

Further comparison of EXP. 1 vs 2 and EXP. 1 vs 3 shows that two etching conditions are necessary for the columnar structure formation: the $\mathrm{C}$-face of $6 \mathrm{H}-\mathrm{SiC}$ rather than the $\mathrm{Si}$-face must be used, and an appropriate constant etching voltage has to be maintained.

\subsubsection{EXP. 1 vs EXP. 2}

Figures 1.10 and 1.11 show SEM images and etching curves, respectively, from the three experiments. In EXP. 1 [Figure 1.10(a-d)], we observe 


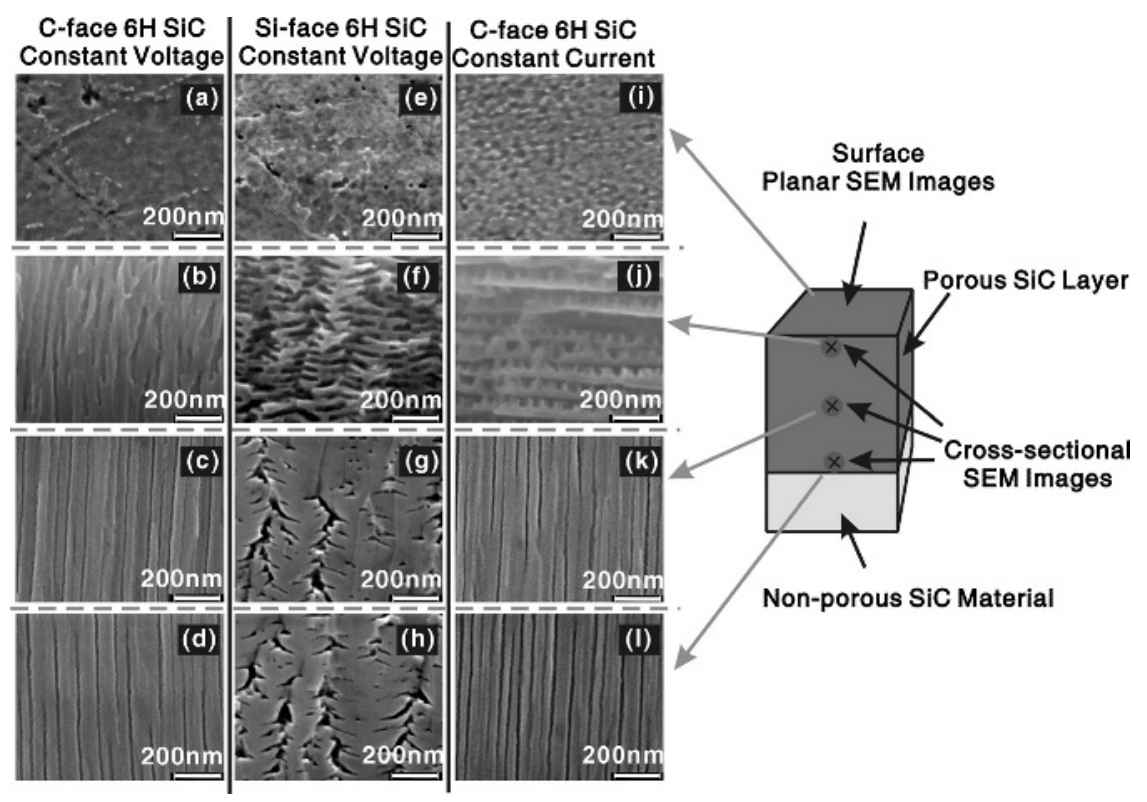

Figure 1.10 Planar and cross-sectional SEM images [34] of (a)-(d) C-face porous $\mathrm{SiC}$ in EXP. 1; (e)-(h) Si-face $6 \mathrm{H}$ porous $\mathrm{SiC}$ in EXP. 2; (i)-(l) $\mathrm{C}$-face $6 \mathrm{H}$ porous $\mathrm{SiC}$ in EXP. 3. Reproduced from Y. Ke, R.P. Devaty and W.J. Choyke, Self-ordered nanocolumnar pore formation in the photoelectrochemical etching of $6 \mathrm{H} \mathrm{SiC,} \mathrm{Elec-}$ trochem. Solid-State Lett., 10(7), K24-K27 (2007). Copyright 2007, with permission from The Electrochemical Society

the formation process of a self-ordered columnar porous structure as described earlier. In EXP. 2 [Figure 1.10(e-h)], even though an identical condition is applied to the Si-face sample, no columnar pore growth is observed. The 'dendritic' pores grow approximately parallel to the $c$-axis with 'branch' diameters of about $20-50 \mathrm{~nm}$. The etching currenttime $(I-t)$ curves of Figure 1.11(a) and Figure 1.11(b) both record a rapid nonexponential drop. After an initial interval, the current density becomes linear on a double log scale and can be fitted to the equation:

$$
I=C t^{\beta} .
$$

For the $\mathrm{C}$-face sample in EXP. 1 , we have $C \approx 510$ and $\beta \approx-0.40$, while for the Si-face sample in EXP. 2, $C \approx 415$ and $\beta \approx-0.43$. The values of $\beta$ are essentially the same within experimental error. The etching on the C-face has a larger constant $C$, corresponding to a larger current density than the etching on the Si-face, but a similar current decay rate. 


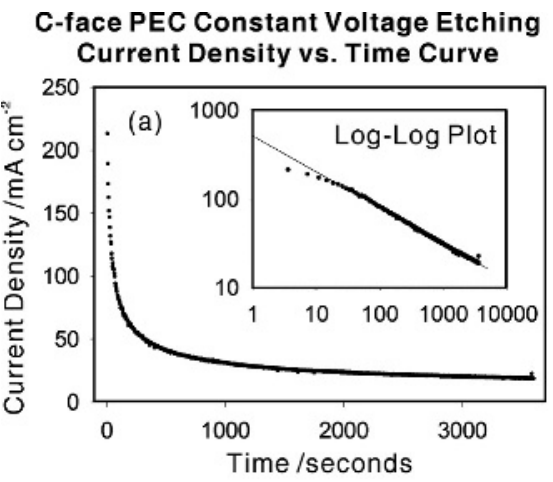

Si-face PEC Constant Voltage Etching

Current Density vs. Time Curve

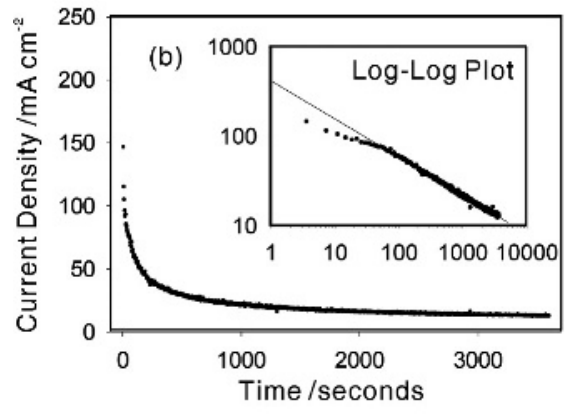

\section{C-face PEC Constant Current Etching} Voltage vs. Time Curve

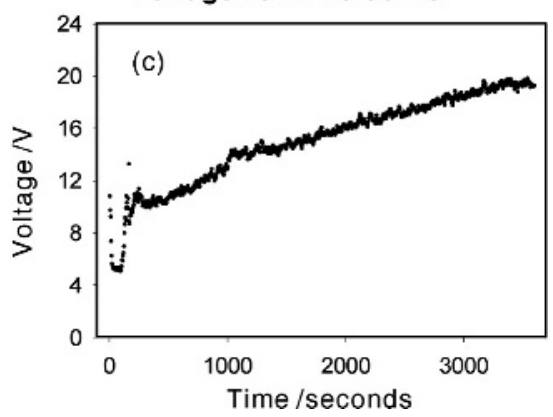

Figure 1.11 I-t curves [34] in (a) EXP. 1 and (b) EXP. 2; (c) $V-t$ curve in EXP. 3. Reproduced from Y. Ke, R.P. Devaty and W.J. Choyke, Self-ordered nanocolumnar pore formation in the photoelectrochemical etching of $6 \mathrm{H} \mathrm{SiC}$, Electrochem. SolidState Lett., 10(7), K24-K27 (2007). Copyright 2007, with permission from The Electrochemical Society 
A higher current density usually reflects a larger electrochemical reaction rate. For the C-face columnar pore growth, the pore geometry and distribution is uniform. As explained earlier, the two-dimensional areal porosity of a columnar porous layer is almost constant below the first few microns of etching. The effective etching area is therefore roughly a constant throughout. Consequently, the high electrochemical reaction rate here indicates a high growth rate of the porous layer thickness at the beginning of the etching process compared with the rate at the end. This behavior is confirmed by etching a C-face sample at constant voltage for $1 \mathrm{~min}$. The resulting $23 \mu \mathrm{m}$ thick columnar porous layer suggests that the average rate of increase of the porous layer thickness for the $1 \mathrm{~min}$ etching is about seven times the rate for $1 \mathrm{~h}$ PECE. However, the reason for this specific etching rate decrease is not clear.

If we compare the current densities at the same etching time in the two experiments, it is found that the current density on the C-face is in general higher than that on the Si-face, which suggests that the reaction rate is higher for the $\mathrm{C}$-face $\mathrm{SiC}$ experiment under the same etching conditions. Current-voltage scan $(I-V)$ measurements were performed on both the $\mathrm{C}$-face and the Si-face of $6 \mathrm{H}-\mathrm{SiC}$ to examine the electrolyte and semiconductor junction properties (Figure 1.12). The results show that, for both the C-face and Si-face, UV illumination in general enhances the current density remarkably. (The 'abnormal' behavior between $\sim 7 \mathrm{~V}$ and $\sim 15 \mathrm{~V}$ for the $\mathrm{C}$-face $\mathrm{SiC}$ scan is not understood.) There are two distinct steps in
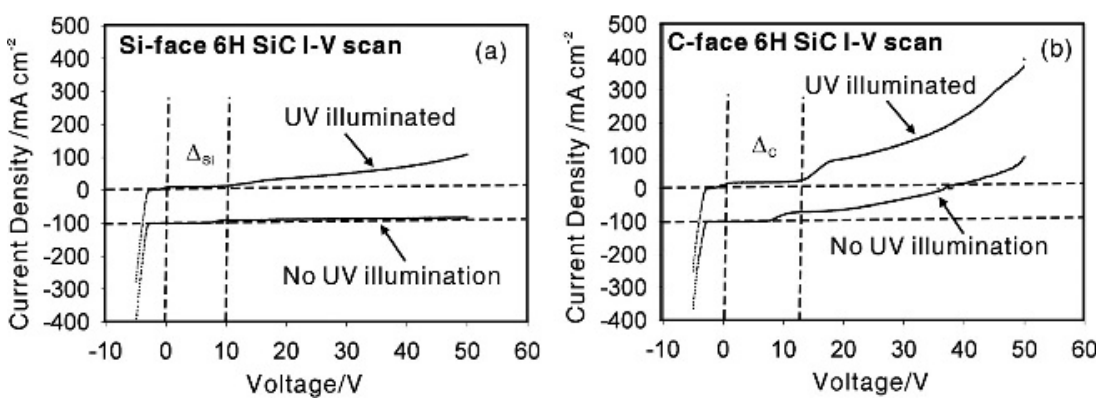

Figure 1.12 $I-V$ scans [34] on the (a) Si-face and (b) C-face of $6 \mathrm{H}-\mathrm{SiC}$ in $10 \% \mathrm{HF}$, $5 \%$ ethanol electrolytic solution at $1 \mathrm{~V} \mathrm{~s}^{-1}$ scan rate. Note that in order to better distinguish the curves recorded with and without UV illumination, the $I-V$ curve without UV illumination is lowered by $100 \mathrm{~mA} \mathrm{~cm}^{-2}$. UV intensity is $600 \mathrm{~mW} \mathrm{~cm}{ }^{-2}$. Reproduced from Y. Ke, R.P. Devaty and W.J. Choyke, Self-ordered nanocolumnar pore formation in the photoelectrochemical etching of $6 \mathrm{H} \mathrm{SiC,} \mathrm{Electrochem.} \mathrm{Solid-}$ State Lett., 10(7), K24-K27 (2007). Copyright 2007, with permission from The Electrochemical Society 
the curves with UV illumination at which the current increases abruptly. These steps are separated by a plateau $\left(\Delta_{S i}\right.$ for the Si-face scan and $\Delta_{C}$ for the $\mathrm{C}$-face in Figure 1.12). Beyond this plateau, the $I-V$ curve for the $\mathrm{C}$-face $\mathrm{SiC}$ shows a larger and more rapidly increasing current density than that for the Si-face. The clear difference between the Si-face and $C$-face $I-V$ curves confirms the dissimilar interface junction properties of the two crystalline faces in a photoelectrochemical process. The larger current density on the C-face provides a faster electrochemical etching rate or oxidation rate than the rate on the Si-face, which is in agreement with the result from wet oxidation experiments [23]. Moreover, the ratio of the porous layer thickness in EXP. 1 to that in EXP. 2 along the $c$-axis is 2.5. This suggests that the PECE more strongly prefers to proceed in the [0001] direction for the C-face than in the [0001] direction for the etching of the Si-face. Therefore, to achieve controllable columnar pore growth (without using avalanche breakdown conditions), PECE must be done in etch preferred directions, in our case, on C-face SiC.

\subsubsection{EXP. 1 vs EXP. 3}

In EXP. 1, in the linear plot [Figure 1.11(a)], after the rapid current density drop at the beginning, the current density stabilizes around $30 \mathrm{~mA}$ $\mathrm{cm}^{-2}$ during most of the etching interval. To check whether this quasiconstant current flow is responsible for the columnar pore formation, we conducted a constant current density experiment (EXP. 3) at $30 \mathrm{~mA}$ $\mathrm{cm}^{-2}$ with all other experimental conditions unchanged. The result shows that the constant current density does not lead to a uniform columnar porous morphology and the resulting porous structure [Figure 1.10(i-1)] is believed to be correlated with the compliance voltage [Figure 1.11(c)], which is applied to maintain the desired current. In the early stage, the porous morphology is triangular [Figure 1.10(j)]. Below this layer, irregular columnar pores are observed. It is only towards the latter stage of etching, when the voltage approaches $20 \mathrm{~V}$, that a relatively ordered and straight columnar porous structure is observed, which resembles the structure fabricated by constant voltage etching in EXP. 1. This result indicates that an appropriate voltage is crucial to form the ordered nano-columnar porous structure. This could also suggest that a specific electric field at the electrolyte/solid interface is necessary to the columnar pore formation, if we assume that the major voltage drop is across the interface during the complete etching process. 
Further comparison of EXP. 1 and 3 on C-face samples shows that the mass loss and the total charge flow are practically identical. However, under the constant voltage condition, the porous layer thickness is about $50 \mu \mathrm{m}$ larger. The constant current condition is a charge supply limited process and the thinner porous structure suggests that the constant voltage etching is likely to be a mass transfer rate limited process. The rate of etching depends on the concentrations of the chemicals and the mass transfer rates of the ions in the electrolyte at the columnar pore tips. These mass transfer rates of the reactant and product ions balance with the hole supply rate from the semiconductor (anodic current) in order to maintain the columnar pore formation. The concentrations of chemicals at the pore tips decrease as the columnar pores become longer. This would explain why columnar pores form much faster at the beginning of the etching.

In EXP. 3, columnar pore growth is optimized when the voltage is around $20 \mathrm{~V}$. A series of SEM images is shown in Figure 1.13 to compare the pore formation when the voltage is lower or higher than $20 \mathrm{~V}$. Figure $1.13(\mathrm{e}-\mathrm{h})$ shows the SEM images of an optimized columnar porous structure under $20 \mathrm{~V}$ as discussed earlier. If $10 \mathrm{~V}$ is applied [Figure $1.13(\mathrm{a}-\mathrm{d})$ ], the porous structure close to the surface has a layered morphology filled

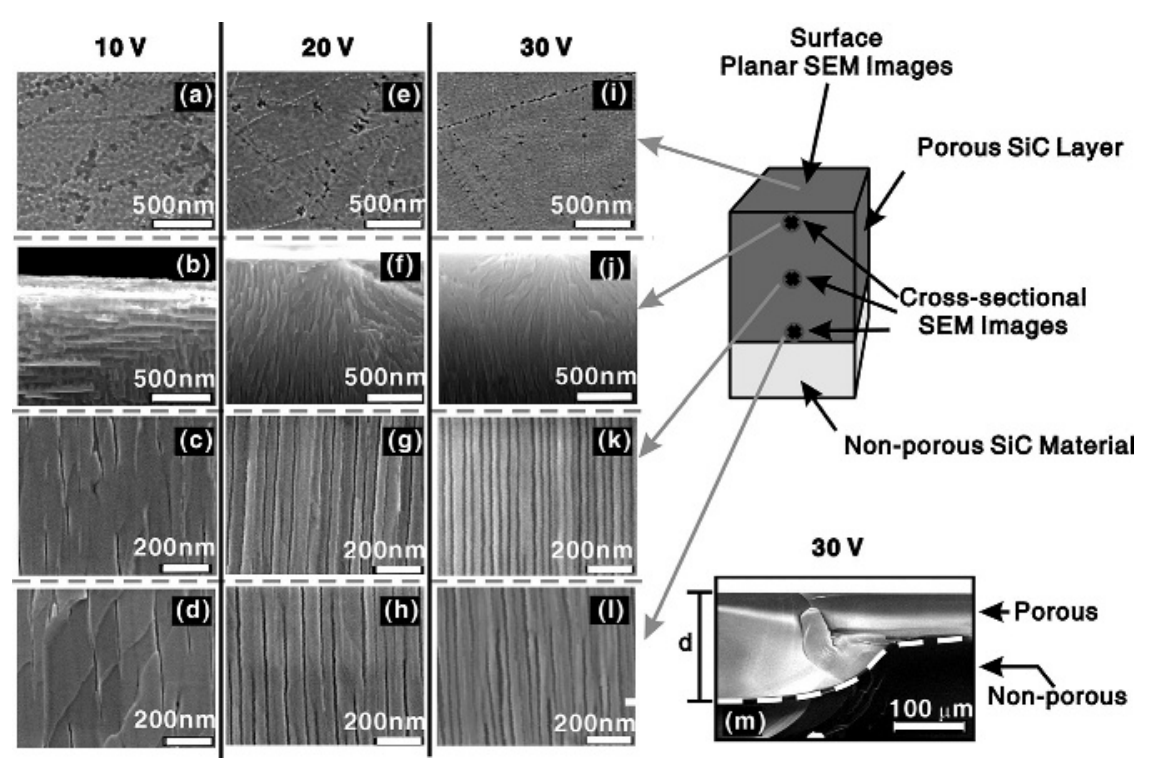

Figure 1.13 Planar and cross-sectional SEM images of (a)-(d) $10 \mathrm{~V}$, (e)-(h) $20 \mathrm{~V}$ and (i)-(l) $30 \mathrm{~V}$ constant voltage etching on C-face $6 \mathrm{H}-\mathrm{SiC}$. (m) Cross sectional image shows the inhomogeneous porous layer thickness of a columnar porous structure fabricated by applying $30 \mathrm{~V}$ voltage 
with triangular shaped pores [Figure 1.13(b)]. The relationship between the underlying pore growth and the surface pores is not obvious. Below this layer, for most of the porous layer, we find a very irregular columnar pore structure. The pore morphology can hardly be recognized as columnar. The sinuous pores go in and out of the cleaved SiC plane and do not show a connected porous network in the SEM cross-sectional image. The final porous structure thickness is about $210 \mu \mathrm{m}$ and the average porosity is 0.1 . Comparing with the $20 \mathrm{~V}$ etching, the porous layer thickness and the overall porosity are both similar.

The $30 \mathrm{~V}$ etching destroys the columnar porous structure in another way. In Figure 1.13(i-1), we can see that the porous structure close to the ambient/porous interface is quite similar to the $20 \mathrm{~V}$ etched porous structure. In the middle of the porous layer, the columnar pores are straight and ordered. The pores are $20 \mathrm{~nm}$ in diameter but the pore density is higher. However, towards the porous/bulk interface, the porous structure becomes irregular. Therefore the columnar porous etching has a depth limitation under $30 \mathrm{~V}$ etching. Most importantly, the thickness of the porous structure is inhomogeneous [Figure $1.13(\mathrm{~m})$ ]. The thinnest part of the structure is about $70 \mu \mathrm{m}$ thick whereas the thickest part is about $200 \mu \mathrm{m}$. It is interesting to see that the maximum thickness remains close to the values obtained in $10 \mathrm{~V}$ and $20 \mathrm{~V}$ etching. However, some part of the porous growth is slower or even stopped at some point. The reason for this large variation of the thickness is not clear. Because of this thickness variation, the porosity of the porous structure can not be evaluated.

The recorded current density-time curves for three different voltages are shown in Figure 1.14. In all three curves we observe a linear decay

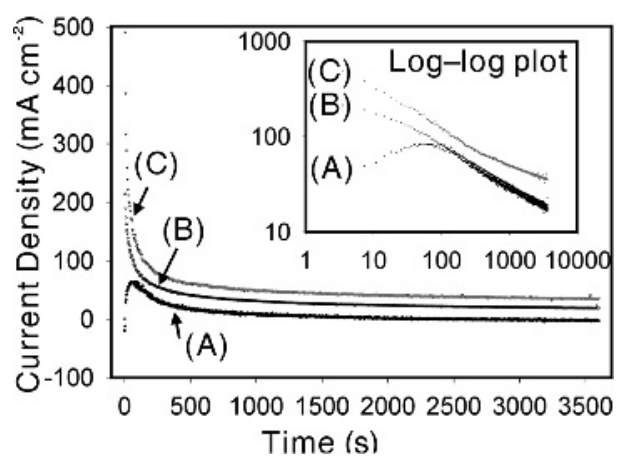

Figure 1.14 Current density vs time curves recorded during constant voltage etching of n-type $6 \mathrm{H} \mathrm{SiC}$ under the voltage (A) $10 \mathrm{~V}$, (B) $20 \mathrm{~V}$ and (C) $30 \mathrm{~V}$. Note that to better distinguish the curves $(\mathrm{A})$ and $(\mathrm{B})$ in the linear plot, curve $(\mathrm{A})$ is lowered by $20 \mathrm{~mA} \mathrm{~cm}^{-2}$. The inset shows the $\log -\log$ plot of the same curves 
section in the log-log plot. If we fit the data using Equation (1.7), we have $C \approx 545$ and $\beta \approx-0.43$ for $10 \mathrm{~V}$ etching, $C \approx 510$ and $\beta \approx-0.40$ for $20 \mathrm{~V}$ etching. For $30 \mathrm{~V}$ etching, there are two linear sections. For the first $500 \mathrm{~s}, C \approx 893$ and $\beta \approx-0.43$, while for the rest of the curve, $C$ $\approx 388$ and $\beta \approx-0.37$. It is interesting to see that the current density curves (A) and (B) overlap after the initial $100 \mathrm{~s}$. The curve fitting results for these two curves are also very close. However, the very different pore morphology suggests that the initial high current flow is important for the columnar pore growth. The curve $(\mathrm{C})$ under $30 \mathrm{~V}$ etching shows a larger current density. The curve has a similar decay constant in the first $500 \mathrm{~s}$, after which the decay rate becomes slower.

Finally, the seemingly necessary constant voltage condition for columnar pore formation might suggest a space-charge-region depletion mechanism for pore wall passivation, which determines the pore wall thickness. The idea that the thickness of the pore walls between two pores is about twice the space-charge-region width is attractive. If we assume that the $20 \mathrm{~V}$ voltage drop is mainly across the space-charge-region of the semiconductor, the width of this space-charge-region is estimated to be about $60 \mathrm{~nm}$ at the cylindrical pore walls, which would suggest an inter-pore wall thickness of about $120 \mathrm{~nm}$. The observed $40 \mathrm{~nm}$ pore wall is not consistent with this estimate. Although we believe that the pore wall is completely depleted, its thickness is apparently not defined by the space-charge-region width. The reasons for the observed $40 \mathrm{~nm}$ inter-pore distance and $20 \mathrm{~nm}$ pore diameter remain unclear and subject to further study.

\subsection{SUMMARY}

In summary, we have succeeded in fabricating porous $6 \mathrm{H}$ - and $4 \mathrm{H}-\mathrm{SiC}$ with various morphologies, among which we have most intensively studied the triangular and the columnar porous morphologies.

Through the investigation of triangular porous $\mathrm{SiC}$, we find that in the PECE of (0001), (1100), and (1210) surfaces of $4 \mathrm{H}-\mathrm{SiC}$ using low current densities (up to $5 \mathrm{~mA} \mathrm{~cm}{ }^{-2}$ ), the shape and the size of the pores remain substantially the same, and the overall macroscopic porous structure is retained. A proposed model accounts for the observed triangular channel pore morphology. The foremost result of the investigation of the planar/triangular porous morphology is the discovery of a new surface which corresponds to the $\{1 \overline{1} 0 \overline{2}\}$ family of planes in $4 \mathrm{H}-\mathrm{SiC}$. 
The successful fabrication of a self-ordered, low porosity (0.1), columnar porous $\mathrm{SiC}$ structure with a pore diameter of about $20 \mathrm{~nm}$ provides a practical porous morphology for potential applications. To control columnar pore growth, voltage rather than current is the decisive etching condition. A proper voltage must be applied to obtain columnar pore formation. Rather than the differences in the photoelectrochemical reactions, it is the fact that the C-face does not act as an etch stop that avoids the problem of branching and favors the formation of straight columnar pores under controllable etching conditions. The further improvement of the pore lattice regularity and control of the pore diameter are challenging as long as the mechanism determining the inter-pore spacing and pore diameter remains unclear. Further understanding of the growth process is challenging. More experimental parameters must be studied in order to model this process.

\section{ACKNOWLEDGEMENTS}

We gratefully acknowledge Albert Stewart, associated with the Materials Micro-Characterization Laboratory of the Department of Mechanical Engineering and Materials Science, for assistance with the electron microscopy conducted during this study. We thank Chris Bowman, supervisor of the clean room at Carnegie Mellon University, for kindly allowing us to use the RIE apparatus and A. Sagar for his help with RIE processing. We also thank CREE, Inc. and II-VI, Inc. for providing us with high quality $4 \mathrm{H}$ - and $6 \mathrm{H}-\mathrm{SiC}$ single crystal wafers. This work is supported by the ONR-DURINT grant N00014-01-1-0715.

\section{REFERENCES}

[1] A. Uhlir, Jr, Electrolytic shaping of germanium and silicon, Bell Sysem. Tech. J., 35, 333-347 (1956).

[2] D.R. Turner, Electropolishing silicon in hydrofluoric acid solutions, J. Electrochem. Soc., 105, 402-408 (1958).

[3] J.W. Faust, Jr, The etching of silicon carbide, in Silicon Carbide: A High Temperature Semiconductor, J.R. O’Connor and J. Smiltens (Eds), Pergamon, Oxford, 1960, pp. 403-419.

[4] Y. Watanabe, Y. Arita, T. Yokoyama and Y. Igarashi, Formation and properties of porous silicon and its application, J. Electrochem. Soc., 122, 1351-1355 (1975).

[5] L.T. Canham, Silicon quantum wire array fabrication by electrochemical and chemical dissolution of wafers, Appl. Phys. Lett., 57, 1046-1048 (1990). 
[6] J.S. Shor and R.M. Osgood, Jr, Broad-area photoelectrochemical etching of n-type beta-SiC, J. Electrochem. Soc., 140, L123-L125 (1993).

[7] J.S. Shor, I. Grimberg, B.-Z. Weiss and A.D. Kurtz, Direct observation of porous $\mathrm{SiC}$ formed by anodization in HF, Appl. Phys. Lett., 62, 2836-2838 (1993).

[8] J.S. Shor and A.D. Kurtz, Photoelectrochemical etching of 6H-SiC, J. Electrochem. Soc., 141, 778-781 (1994).

[9] J.S. Shor, L. Bemis, A.D. Kurtz, I. Grimberg, B.Z. Weiss, M.F. MacMillan and W.J. Choyke, Characterization of nanocrystallites in porous p-type 6H-SiC, J. Appl. Phys., 76, 4045-4049 (1994).

[10] M.F. MacMillan, R.P. Devaty, W.J. Choyke, D.E. Goldstein, J.E. Spanier and A.D. Kurtz, Infrared reflectance of thick p-type porous SiC layers, J. Appl. Phys., 80, 2412-2419 (1996).

[11] J.E. Spanier and I.P. Herman, Use of hybrid phenomenological and statistical effective-medium theories of dielectric functions to model the infrared reflectance of porous SiC films, Phys. Rev. B, 61, 10437-10450 (2000).

[12] S.E. Saddow, M. Mynbaeva and M.F. MacMillan, Porous SiC technology, in Silicon Carbide: Materials, Processing and Devices, Zhe Chuan Feng and Jian H. Zhao (Eds), Taylor and Francis, New York, 2004, Chapter 8, pp. 321-385.

[13] Y. Shishkin, Y. Ke, R.P. Devaty and W.J. Choyke, A short synopsis of the current status of porous SiC and GaN, Mater. Sci. Forum, 483-485, 251-256 (2005).

[14] Y. Shishkin, W.J. Choyke and R.P. Devaty, Photoelectrochemical etching of n-type 4H silicon carbide, J. Appl. Phys., 96, 2311-2322 (2004).

[15] E.J. Garboczi, D.P. Bentz and N.S. Martys, Digital images and computer modeling, in Methods in the Physics of Porous Media, Po-zen Wong (Ed.), Experimental Methods in the Physical Sciences 35, Academic Press, San Diego, 1999.

[16] Y. Shishkin, Y. Ke, R.P. Devaty and W.J. Choyke, Fabrication and morphology of porous p-type SiC, J. Appl. Phys., 97, 044908 (2005).

[17] M.I.J. Beale, N.G. Chew, M.J. Uren, A.G. Cullis and J.D. Benjamin, Microstructure and formation mechanism of porous silicon, Appl. Phys. Lett., 46, 86-88 (1985).

[18] A.O. Konstantinov, C.I. Harris and E. Janzén, Electrical properties and formation mechanism of porous silicon carbide, Appl. Phys. Lett., 65, 2699-2701 (1994).

[19] O. Jessensky, F. Müller and U. Gösele, Microstructure and photoluminescence of electrochemically etched porous SiC, Thin Solid Films, 297, 224-228 (1997).

[20] A. Bauer, J. Kräußlich, L. Dressler, P. Kuschnerus, J. Wolf, K. Goetz, P. Käckell, J. Furthmüller and F. Bechstedt, High-precision determination of atomic positions in crystals: the case of 6H- and 4H-SiC, Phys. Rev. B, 57(5), 2647-2650 (1998).

[21] E. Rauls, J. Elsner, R. Gutierrez and Th. Frauenheim, Stoichiometric and nonstoichiometric (1-100) and (11-20) surfaces in 2H-SiC: a theoretical study, Solid State Commun., 111, 459-464 (1999).

[22] E. Burstein and P.H. Egli, The physics of semiconductor materials, in Advances in Electronics and Electron Physics, L. Marton (Ed.), Academic Press, Inc., New York, 1955, Vol. VII, p. 43.

[23] L. Muelhoff, M.J. Bozack, W.J. Choyke and J.T. Yates, Jr, Comparative oxidation studies of SiC(000-1) and SiC(0001) surfaces, J. Appl. Phys., 60, 2558-2563 (1986).

[24] Y. Shishkin, E. Oborina, A. Maltsev, S.E. Saddow and A.M. Hoff, Oxide of non-basal quasi-polar 6H-SiC surfaces, J. Phys. D: Appl. Phys., 39, 2692-2695 (2006).

[25] Y. Shishkin and O. Kordina, Bulk growth of $6 \mathrm{H}-\mathrm{SiC}$ on non-basal quasi-polar surfaces, J. Cryst. Growth, 291, 317-319 (2006). 
[26] U. Starke, W.Y. Lee, C. Coletti, S.E. Saddow, R.P. Devaty and W.J. Choyke, SiC pore surfaces: Surface studies of 4H-SiC(1-102) and 4H-SiC(-110-2), Appl. Phys. Lett., 88, 031915 (2006).

[27] S. Frey, M. Kemell, J. Carstensen, S. Langa and H. Föll, Fast pore etching, Phys. Status Solidi A, 202(8), 1369-1373 (2005).

[28] H. Föll, M. Christophersen, J. Carstensen and G. Hasse, Formation and application of porous silicon, Mater. Sci. Eng. R 39, 93-141 (2002).

[29] H. Masuda and K. Fukuda, Ordered metal nanohole arrays made by a two-step replication of honeycomb structures of anodic alumina, Science, 268, 1466-1468 (1995).

[30] K. Nielsch, J. Choi, K. Schwirn, R. Wehrspohn and U. Gösele, Self-ordering regimes of porous alumina: the $10 \%$ porosity rule, Nano Lett, 2(7), 677-680 (2002).

[31] S. Langa, M. Christophersen, J. Carstensen, I.M. Tiginyanu and H. Föll, Single crystalline 2D porous arrays obtained by self organization in n-InP, Phys. Status Solidi A, 197(1), 77-82 (2003).

[32] H. Föll, S. Langa, J. Carstensen, M. Christophersen, and I.M. Tiginyanu, Pores in III-V semiconductors, Adv. Mater. 15(3), 183-198 (2003).

[33] Y. Ke, F. Yan, R.P. Devaty and W. J. Choyke, Columnar pore growth in n-type $6 \mathrm{H}$ SiC, Mater. Sci. Forum, 527-529, 739-742 (2006).

[34] Y. Ke, R.P. Devaty and W.J. Choyke, Self-ordered nanocolumnar pore formation in the photoelectrochemical etching of $6 \mathrm{H} \mathrm{SiC,} \mathrm{Electrochem.} \mathrm{Solid-State} \mathrm{Lett.,} 10$ (7), K24-K27 (2007).

[35] Y. Ke, C. Moisson, R.M. Feenstra, R.P. Devaty and W.J. Choyke, A comparison of various surface finishes and the effects on the early stages of pore formation during high field etching of SiC, Mater. Sci. Forum, 527-529, 743-746 (2006). 
